\title{
LCA's theory and practice: like ebony and ivory living in perfect harmony?
}

\author{
Martin Baitz • Stefan Albrecht • Eloise Brauner • Clare Broadbent • Guy Castellan • \\ Pierre Conrath • James Fava • Matthias Finkbeiner • Matthias Fischer • \\ Pere Fullana i Palmer • Stephan Krinke • Christian Leroy • Oliver Loebel • \\ Phil McKeown • Ivo Mersiowsky • Bernhard Möginger • Marcus Pfaadt • \\ Gerald Rebitzer • Elmar Rother • Klaus Ruhland • Aafko Schanssema • Ladji Tikana
}

Received: 11 July 2012 /Accepted: 11 July 2012 /Published online: 31 July 2012

(C) The Author(s) 2012. This article is published with open access at Springerlink.com

\section{Introduction}

Life cycle assessment (LCA) is recognized as a trustworthy, scientific while understandable approach to address the environmental sustainability of human activities. It is applied for multiple uses in internal and external information supply and for decision support. However, LCA application in

M. Baitz $(\bowtie)$

PE INTERNATIONAL AG,

Hauptstrasse 111-113,

70771 Leinfelden-Echterdingen, Germany

e-mail:m.baitz@pe-international.com

\section{S. Albrecht}

University of Stuttgart (LBP),

Hauptstraße 113,

70771 Leinfelden-Echterdingen, Germany

\section{E. Brauner}

PE INTERNATIONAL AG,

Hauptstrasse 111-113,

70771 Leinfelden-Echterdingen, Germany

\section{Broadbent}

World Steel Association,

Rue Colonel Bourg 120,

1140 Brussels, Belgium

G. Castellan

PlasticsEurope Association,

Avenue E. Van Nieuwenhuyse 4, Box 3, 1160 Brussels, Belgium

P. Conrath

EDANA Association,

Avenue Herrmann Debroux 46,

1160 Brussels, Belgium

J. Fava

Five Winds,

344 Boylston Street,

Boston, MA 02116, USA practice must fulfill three basic criteria: (1) It must be reliable in order to ensure the credibility of information and results generated, (2) it must fit into existing information routines and practices in business to ensure applicability, and (3) it must provide quantitative and relevant information to inform decision makers. Over the last two decades, LCA methodology and related data have become a suitable and professional

\section{Finkbeiner}

Sustainable Engineering, Technical University of Berlin,

Strasse des 17. Juni 135,

10623 Berlin, Germany

M. Fischer

Fraunhofer Institute of Building Physics (IBP),

Nobelstr. 12,

70569 Stuttgart, Germany

P. Fullana i Palmer

UNESCO Chair in Life Cycle and Climate Change at ESCI-UPF,

Pg. Pujades, 1,

Barcelona, Spain

S. Krinke

Volkswagen AG,

Brieffach 1774,

38436 Wolfsburg, Germany

C. Leroy

European Aluminum Association,

Avenue de Broqueville, 12,

1150 Brussels, Belgium

O. Loebel

PU Europe Association,

Av. E. van Nieuwenhuyse 6,

1160 Brussels, Belgium

P. McKeown

Safety and Environmental Assurance Centre (SEAC), Unilever PLC,

Colworth Park Sharnbrook,

MK44 1LQ Bedfordshire, UK 
tool to address and beneficially influence environmental aspects of sustainability of virtually all anthropogenic activities. LCA is a field of scientific research but is also a business of growing importance. Many users from the academia, industry, or consultancy all over the world apply LCA in scientific, industrial, agricultural, societal, or political processes and use their findings. The diverse group of authors is proactively working in the LCA field, in methodology development, data provision, data compiling, or product optimization and communication. The authors' respective affiliations apply, assess, and support LCA; they use it as a decision support and communication tool in their organizations or along value and process chains and to discuss with stakeholders. Dealing with technical boundaries and identifying realistic potentials based on sound scientific information is the foundation of their LCA work.

\section{Aim}

This editorial aims to improve cooperation in the use of LCA in both theory and practice. The successful

I. Mersiowsky

DEKRA Consulting GmbH,

Handwerkstraße 15,

70565 Stuttgart, Germany

\section{B. Möginger}

Bonn-Rhein-Sieg University of Applied Science, von-Liebig-Str. 20,

53359 Rheinbach, Germany

\section{Pfaadt}

Wacker Chemie AG,

Burgkirchener Str. 1,

84489 Burghausen, Germany

G. Rebitzer

Value Innovation Centre, Amcor Flexibles Kreuzlingen AG,

Badische Bahnhofstr. 16,

8212 Neuhausen, Switzerland

\section{E. Rother}

Evonik Industries AG,

Rodenbacher Chaussee 4,

63457 Hanau-Wolfgang, Germany

K. Ruhland

Daimler AG,

71059 Sindelfingen, Germany

A. Schanssema

VMK Kunststofverpakkingen,

Loire 150 ,

2491 AK Den Haag, The Netherlands

L. Tikana

Deutsches Kupfer Institut (DKI),

Am Bonneshof 5,

40474 Düsseldorf, Germany development of LCA requires, on one hand, an influx of new ideas and harmonized methodologies, and on the other hand, thriving and credible application. The authors share the implications of LCA in daily businesses and practice and aim to nurture and strengthen the interfaces between scientific findings and application. Working together to encourage a broader application of "good practice" LCA in industry as well as strengthening scientific LCA work towards "applicable science" will develop and reinforce professional LCA work and technical implementation in the academic and business arena. This article is written with a primary focus on industrial applications and research in applied science and with less emphasis on specific governmental applications.

\section{Setting the scene}

To catalyze cooperation and reduce the risk of improper application of LCA in business, the authors outline ten overarching aspects to set the scene:

1. Industry needs to understand new scientific methods and academia needs to understand the practical boundaries that exist where these new methods will be applied. Industry needs comprehensive, well-accepted, and consistent LCA methods with a high credibility to implement into business and communication processes, while academia needs industry to apply their new findings.

2. Most of the LCA work published is from academia. Most LCA work conducted, compiled, and put into practice in industry has not been published due to commercial implications.

3. A fruitful exchange of detailed supply chain information within the "(non-public) secured B2B ${ }^{1}$ environments" exists today and supports LCA application in business.

4. Newcomers to the growing LCA community should be aware that B2B, B2C, ${ }^{2} \mathrm{~B} 2 \mathrm{G}^{3}$ and academic networks do exist, albeit with different goals, and should understand the similarities and differences in these networks in order to liaise between the different groups.

5. The primary aim of LCA in business is to improve products and processes; this relies upon a foundation of sound and applicable (scientific) methods.

6. The primary aim of LCA in academia is to improve methods; this relies upon application in case studies as validation for practical implementation.

7. $\mathrm{B} 2 \mathrm{~B}$ and value chain information exchange is essential for LCA work.

\footnotetext{
${ }^{1} \mathrm{~B} 2 \mathrm{~B}$ : business to business

${ }^{2} \mathrm{~B} 2 \mathrm{C}$ : business to customer

${ }^{3} \mathrm{~B} 2 \mathrm{G}$ : business to government
} 
8. LCA information that is used for communicating to customers (often non-LCA experts) must be clear and understandable.

9. LCA practitioners must acknowledge the distinctions between "latest science" and "good practice".

10. Professional practice demands data that reflect the technical reality and is therefore suitable for various sustainability applications (LCA, EPD, ${ }^{4}$ sustainability assessments, footprinting approaches, etc.)

These aspects frame the discussions on LCA applications in business practice, bearing in mind that it is used not only for environmental improvement of products and processes but also for cost reduction, sustainable development strategies, risk management, defining competitive advantage and increasing (sustainable) revenue.

\section{LCA as a business case}

The following section illustrates the authors' position, addressing concrete implications of LCA in daily business. These examples do not claim to be exhaustive and are open for comment.

Success factors for LCA in industry If we understand LCA as a business imperative, we must be able to clearly present its success factors. In most companies and associations where it is applied today, LCA is no longer a purely "voluntary" or "freestyle" activity; rather, it is seen as a fundamental activity of the organization. It is recognized as the best available methodology to investigate environmental sustainability performance in a reliable and transparent way. It can be used for communication, both along the value chain and throughout one's own organization. It supports and helps to cross-check both research and development and strategic decisions. LCA in practice must be time-efficient and investment costs and resource availability must be accounted for. Therefore, it is essential that data generation/acquisition and execution of LCA is efficient. This includes the ability to acquire industryaverage data from multiple sites/companies and effective opportunities to close data gaps in an optimal tradeoff of precision and effort. Several approaches and methods to close data gaps are discussed in theory; however, practical engineering expertise and a meaningful exchange of information between industry, consulting, and science are fundamental.

In industry, it is imperative that LCA results and the underlying data be converted into a technical conclusion, the nature of which is determined by the recipient of the result (e.g., product engineers, marketing, suppliers, or consumers). LCA is applied for quantitative environmental

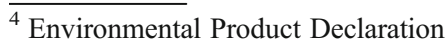

management and should reflect the industrial reality adequately. It is important that hot spots for product optimization can be identified along the entire value chain, based on a common understanding of the chain links. Standardization of procedures is the key to ensure a common interpretation of results within the chain links. LCA is considered an internationally accepted method and a firm basis for a dialogue with internal and external stakeholders.

It is common practice in industry to benchmark one's own processes and products against the competition - commonly on a cost basis. It must be understood that following presentation of an LCA study, non-LCA experts will ask for an evaluation of the results within the competitive landscape. Although a specific result may not be simply "compared" with a database value - it is common practice for internal use in industry.

Aside from the use of LCA as an internal planning tool within a single organization, another potential lies in connecting partners along the value chain. By collaborating on an LCA, suppliers and customers strengthen their relationship, glean valuable insights in markets and their success factors, and enhance an overall exchange of experiences. This fosters innovation.

Single scores and footprinting approaches seem simple to understand for a wider audience, can deem benefits for communicating results, and may demand somewhat less data. Nevertheless, one of the key advantages of using LCA is being able to communicate credibly and comprehensively. If at all, most LCA practitioners only consider using (single) score approaches when they have been able to see the range of impacts from the standard LCA approach. If adequate LCI foreground and background data are available - as is the case for many companies and organizations - the potential time saved by using a single impact approach can be insignificant. Single impact methods can be produced from any LCA simply by "hiding" other impacts. When the market is asking for indicators in specific impact categories, it is much better to provide information through a sound LCA than to wait for someone from outside the LCA community to provide whatever result based on non-LCA methods.

It is mainly method developers and scientists who think weighting is so crucial for application. The real-world users who apply LCA for decision making usually appreciate being able to understand trade-offs and the associated learning's involved rather than looking for black and white solutions. Professional decision makers are used to make decisions in uncertain, multidimensional situations. They do not trust single score values. Many decision makers who use LCA repeatedly on their products realize that they do not need weighting, many do not even think or discuss on impact assessment level. Having done LCIA a few times, they know which inventory flows or rather process data determine the performance for a particular product. In some cases, like communication of environmental information of 
products to consumers, aggregation of LCA results into a single score may be relevant, provided it has reached a level of consensus in a category of products, and that it is possible to trace back the results in a transparent manner.

Financially speaking, today LCA even influences the economics - e.g., the shareholder value - of organizations directly as structured and continuous LCA use are increasingly acknowledged as a positive aspect in company ratings.

LCI methods-goal and scope drives necessary data Robustness of results is very important in applied LCA. Potentially multi-million Euro investments or costly design improvements can be decided only on very robust and quality-assured results. The more assumptions are put into a LCA model the, vaguer the result will be. It is therefore in the interest of any practitioner to have robust and reliable results to justify their decisions.

Attributional LCA describes the environmentally relevant flows to and from a product or process and is therefore the backbone for robust results in practice. Changes in results relate directly to modifications of the technical processes under analysis, uncertainties, or variations in results are understandable and quantifiable over parameter variations and future trends can be addressed via suitable technical scenarios.

Consequential LCA describes how relevant environmental flows will change in response to possible decisions. The potential of consequential LCA $\mathrm{L}^{5}$ must be validated against the associated risks of virtually extended systems and growing uncertainties due to assumptions of related or possible consequences. Consequential LCA is an interesting idea, but for robust application, the main challenge is the reliance on predicted market effects. Predicting the future market behavior is not straightforward-some say it is like looking into a crystal ball-yet uncertain market predictions determine the results of consequential LCAs. Another problem is that consequential LCAs do not address accountabilities appropriately and can provide misleading incentives. However, many important decisions in the economic field (product launch and policy implementation) are actually taken on the basis of prospective studies composed of assumptions and bets on behavior and interactions between stakeholders, on technological breakdown or on threshold effects. LCA and proper scenarios were already used to enlighten these investigations with the environmental dimension long before the consequential approach was developed. The underlying assumptions should always be included, but especially with the results of any consequential LCA.

Input-Output ${ }^{6}$ LCA approaches and other adaptive approaches aim to acquire data comparatively easily where

\footnotetext{
$\overline{5 \text { and/or related }}$ approaches

${ }^{6}$ Input-output tables (I/O) originally focus on monetary exchange figures between generic industry sectors
}

process data are missing. They are often used by practitioners with no access to real-world product and process data or by researchers who are not familiar with the characteristics of a certain industry. However, these approaches also bear the risk of yielding relatively misleading results. These data are not sensitive to company-specific information, since for the most part, average sector or branch data are reported. Hybrid LCA approaches combine I/O table data with process LCA and aim to overcome the problems that prevent a meaningful use of $\mathrm{I} / \mathrm{O}$ data in optimization of specific products or processes in companies. But the efforts to supersede process LCA data compilation can bear nonquantifiable risks and uncertainties since the user often does not know which parts of the data are from sector averages and which are from specific processes. Currently, there is much talk about consequential LCA and input/output-LCA, but little real-world application of it. Scientific research should not lose sight of improving attributional LCA and how to apply it efficiently.

Consequential LCA or other adaptive LCA approaches should therefore not be discussed as an alternative to attributional, process-based LCA in practice, but depending on goal and scope as optional or complementary in sensitivity analysis, for macro-economic scale-up of results or for prospective studies.

Immature $L C I A^{7}$ approaches and their implications on LCI data LCA relies on improvements in LCIA; however, a new single impact method or even a new methodology (as a set of methods) does not automatically mean an improvement in LCA. New LCIA approaches require sufficient testing, such as benchmarking against existing approaches, before they can be applied in industry. It is the responsibility of the developer to explain the practical value of the new approach, if he or she is striving for application and implementation, rather than leaving this up to the user's judgment or creativity.

Using a new LCIA approach can often lead to different LCA results despite having used the same LCI data. Developers must understand that if their approach is to be applied in practice, they need to help users by providing them with the distinct reasons and explanations for these differences. This should not be seen as a hurdle for developing new LCIA approach, but rather as a safeguard for decisions made using LCA with established and tested methods or methodologies.

To avoid unnecessary costs and erroneous or unsound decisions, LCIA methods or methodologies must be able to produce stable results or understandable improvements. Decisions made in product $R \& D^{8}$ today should still be sound a couple of years into production.

\footnotetext{
${ }^{7}$ LCIA: life cycle impact assessment

${ }^{8} \mathrm{R} \& \mathrm{D}$ : research and development
} 
Suitable scenarios that quantify the relevance and bandwidth of possible technical or methodological variations within a life cycle are often more meaningful than a neverending (and never fully justifiable) search for the ultimate truth or ideal approach. Water impacts are a good example for a "new kid on the block" in LCA. Regional and local water topics are to be addressed, while complexity must stay manageable. However, for water life cycle impacts to be applied in practice, an applicable (and tested) approach must be available to fit all relevant branches and regions and the LCI data must be adapted in the same consistent way. Isolated sector approaches could cause inconsistent data and results related to real-life process chains, which tend to cross many sectors and branches.

The consistency and comprehensiveness of a new LCIA approach - and its new set of characterization factors to replace older ones - must be checked against existing data. This should happen before recommendation for application in practice to avoid confusion and misinterpretation. There has to be a good reason if an established impact category characterizes 50 emissions and a newly developed "better" one only 10 . This reason should be clearly communicated so that users can explain it to their stakeholders.

The frequency of implementing new characterization factors or updating methods is a knife-edge; when LCA results change too often due to new approaches (but same LCI data), their credibility diminishes.

Unit processes and aggregated processes For some LCA stakeholders, there still seems to be a debate on "unit processes versus aggregated processes": In practice, there is no right or wrong, but simply a more suitable or less suitable approach depending upon what questions are being asked.

Depending on the goal, both unit processes and aggregated processes are needed in applied LCA. It is rather a question of what needs to be achieved, in which time frame and with which representation. One should pose the questions: "Which foreground system processes are needed to make my model specific to my technological situation and which are needed in the background system to represent the rest of the world adequately?"

Unit processes are often company or technology specific. Therefore, the sole existence of one unit process for a certain material, fuel, or chemical may not guarantee appropriateness or representativeness without adequate technological meta-information. The foreground system often calls for company- or supplier-specific information to make it relevant for the actual case.

Company- or supplier-specific information is often unsuitable for public use or publication. Competition, patent, and anti-trust regulations often call for a protection of such data to avoid public or competitive alignment. Aggregated processes therefore facilitate the use of up-to-date unit process data in LCA data provision, which would otherwise not be available to LCA users.

Using aggregated processes enables LCA practitioners to produce results relatively quickly. Moreover, aggregating data makes it possible to represent suitable technology mix data, where several different supply routes exist for one product on the market. Aggregated processes are needed in background systems to bring meaningful information along the value chain. They are also used to reflect the interconnected nature of many supply chains, industrial networks, and integrated sites properly. Processes are often connected in specific ways, and recombining them can cause over or underestimation or just be incorrect.

To be transparent, it is not necessary to disclose sensitive company or industry-specific data or to infringe confidentiality regulations. Suitable documentation of the modeled processes and technology routes and the relation of technology upstream routes to specific production technologies are of importance and serve the legitimate call for transparency well. Random connection of a process chain can lead to completely unrealistic figures if certain precursor technologies simply do not fit to the reality of downstream production technologies. As such, unit process data should be "industry-borne" or "industry-validated" and must be interconnected to the correct choice of up and downstream unit processes. These unit processes must not necessarily be public domain.

Thus the "unit process versus aggregated process" debate is rather a myth than of practical relevance and aggregated data are without alternative in applied LCA, side by side with company or technology-specific unit process data. If representative and actual industrial situations are of importance, then the "premier league" of versatile LCA data for the general public is industry-borne information that has been compiled by suitable organizations with access to company or industry data and is validated by neutral third parties. Dynamic developments in industrial value chains means that using information from literature sources or other public data that have no suitable validation can pose a high risk of inappropriate results.

Consistency of data and methods When data sources are chosen randomly and mixed, it is difficult to distinguish between real technical differences and differences in background data. The differences in background data for "serious" databases are comparatively easy to identify; either by consulting the existing documentation and/or contacting the respective data provider. If the system is compiled carefully and consistently, interpretation can focus on the technological aspects of the foreground system, the relevance of background systems and implications of both on the total system.

The notion that sometimes the most relevant impacts originate from outside the foreground data, should not 
mislead to the conclusion that generic background data are inferior and stakeholders are forced by default to collect primary data from their suppliers. Using validated background datasets can achieve good quality, even though it is a different "mode" of doing LCA compared to their own specific collection of activity data. But "patchwork" data from unidentified, fuzzy and outdated sources or origins can cause multiple risks in practice, such as:

- double-counting of emissions in groups like VOC, TOC, etc.;

- disaggregation of different flows for same emissions (e.g., $\mathrm{NO}_{x} / \mathrm{NO}_{2} / \mathrm{NO}$ );

- $\mathrm{CO}_{2}$ uptake or storage partly modeled and partly not;

- biogenic and non-biogenic emissions partly separated and partly not;

- mix of company-specific technology routes with random or "virtual" supply chains;

- use of different energy models, which can lead to a comparison of energy modelsrather than to a comparison of the technologies.

The importance of LCI method consistency such as pure allocation, pure substitution, or pure system expansion is often overestimated. In practice, the main objective is to mirror the reality of the technical process chains in the most consistent way and quantify the relevance of options, rather than to simply stick to one method. Besides, the choice of LCI method should not dominate the result; if it does, it is probably not a solid basis for decision making and needs further clarification anyway or other alternatives should be tested.

It is important to be able to explain the changes in LCI data based on facts and changes in technology and value chains. Changes based solely on methodological choices require additional critical discussion in any case before they can provide decision support. An "all in one approach" or applying "stiff methodological rules" is only suitable for a distinct decision context of distinct products in distinct life cycle situations. If the decision context, products, or life cycle situations change, the appropriateness and applicability of the methodological rules to reflect the technical reality must be examined.

Regionalization Regionalization is an extremely relevant issue in applied LCA due to the international coverage of production sites and supply chains. Many national and international programs call for region-specific rules and requirements for data.

Regionalization in LCA is very often understood as simply checking, benchmarking, or matching known processes from a base region to another region. However, truly regionalizing technologies requires engineering know-how and an understanding of the target region rather than smart methods and software support. Regionalization is also a matter of interaction between LCI and LCIA; regionalization in LCI demands for suitable impact assessment evaluation and regionalization in LCIA methods demands a respective basis of regionalized LCI data.

The role of technology and region-specific upstream supply chains is a key. Realistic regionalization is as dynamic as changing markets and needs constant controlling and adjustment. Multinational companies and their market or business-development sometimes influence changes in regional production technologies far more than pure geography.

Regionalization needs a flexible approach which can be individually intensified if the goal and scope call for it. The first and simplest step is to transfer (unit process) information to another country and solely adapt the energy supply. This step alone might be sufficient if process emissions, yield, and engineering circumstances play no relevant role and the result is dominated by the energy use anyway; however, this is often not the case. A second step is to adapt the important upstream processes with regional data and a third step is to check or collect information on the specific target production technology used in the region to adapt unit process information accordingly. This third and most relevant step - while most time intensive-is about collecting and validating industry data in the regional networks of the key branches. This premium approach to acquire regional data is not always suitable. However, if having credible results is critical and (costly) decisions are to be supported, the effort invested for this full-scale approach is quickly compensated. In this context, one should also note that there are cases-e.g., in commodities-where regionalization may even lead to incorrect results. In these instances, where the specific supply mix constantly changes and no chain-ofcustody exists, generic data related to the mix of production are usually much more appropriate.

In the end, the goal and scope will determine the preferred intensity of regionalizing data. But in practical application, realistic regionalization will probably always call for more than just a software-supported switch of energy boundaries.

Rely on reality New scientific methods are important, but if they aim to be applied in practice they should have one goal: To describe the reality or possible trends in a more adequate or comprehensive way than existing methods do. Further they should be comprehensible not only for the developer but also for users. If this is not the case, the method is not adding value and should not be applied, as it would cause unnecessary cost and would not improve the user's information.

Developers of new methods can claim that their method is an improvement, which is always welcomed by 
practitioners; however, the new method is most successful when stakeholders that operate, facilitate, and apply the real processes have the chance for testing its feasibility in application. This is especially true where the new methods aim to influence existing production technology or processes. The new method must be able to transition smoothly into the existing day-to-day business of intra-company and intercompany data compilation and exchange. In practice, LCA is all about description, improvement, and communication of the environmental performance of real products or realistic new product concepts; therefore specific results need specific data and relevant results need relevant data. Involvement of LCA-active companies in validation and method verification might be a promising approach. Sound science is a very important basis for any new LCA development, but it is not the exclusive tiller man in decision support.

A separation of methodology development and LCA application is suggested to promote LCA in industry (Fig. 1).

There is both synergy and tension between the scientific development and the application of LCA methodology. It is the combination of research and application that often leads to disappointment in the capabilities and usefulness of LCA. With this in mind, the usual focus on methodological discussions must be critically evaluated. The choice of the "best" methodology is not necessarily the crucial point for a successful project. A meaningful LCA application, which adapts pure methodology to the real world, copes with real industries, with real products, with real data, with conflicting interests and a large number of stakeholders from government, industry, civil society, and academia is of more value. Of course, progress in LCA research is important and necessary. It is the basis for application. However, applying pure research results is not realistic.

Frequency of updating database information and industrial processes The issue of updating of data is fueled by two critical factors: relevancy of changes in technology and frequency of changes. The right timing of data updates is essential. Some processes hardly change at all over decades, others change annually. The user should not be forced to spend time and money on irrelevant updates.

On account of costs, efficiency, and stability of relevant results, updates of LCI technology data should only be performed if relevant changes or improvements have taken place. It is important to be able to explain the changes in LCI data based on facts and tangible changes in technology/ supply chain and not solely point towards changes in methodology. Changes in methodology should - if at all — rather lead to additional datasets (which can be implemented or neglected by the user depending on the goal and scope).

An increasing challenge in industry is the demand for regular reporting along the value chain, e.g., to customers. Updates of databases can cause changes in LCA results without any change in the actual process data, because raw materials and energy systems upstream have been updated. These changes must be documented properly so that organizations can explain their own achievements and the changes caused by the upstream and background systems.

Conversely, necessary updates must not come too late. If processes or process chains change, this information should be conveyed to the LCA community as soon as possible and older processes should be removed or updated. The fadingout or withdrawing of outdated data reduces the risk of inappropriate LCA results.

\begin{tabular}{|l|c|c|}
\hline & Methodology Development & LCA Application \\
\hline Primary Goals & $\begin{array}{c}\text { Good / Improved Methods } \\
\text { Good / Improved Science } \\
\text { Good / Improved Business }\end{array}$ \\
\hline Secondary Goal & $\begin{array}{c}\text { Reduction of environmental interventions: energy demand, Green-House-Gases, } \text { etc. } \\
\text { "You can reduce } \mathrm{CO}_{2} \text { with a "bad' and scientifically poor LCA, if it is applied." }\end{array}$ \\
\hline Past & "You can NOT reduce $\mathrm{CO}_{2}$ with a 'good' and scientifically brilliant LCA, if it is NOT applied." \\
\hline Future & "poor science and poor application" \\
\hline
\end{tabular}

Fig. 1 Separation of methodology development and LCA application for good science and good application 
Verification of LCI datasets and the critical review of LCA data in studies are both essential for checking, validating, and reviewing data, results, and conclusions. A very effective review of datasets occurs when they are used and benchmarked in practice by practitioners that know the process or supply chain and can judge the results with technological expertise.

The future will likely bring more governance concerning the format, type, and documentation of data, certain practitioners might become accredited and data will only be accepted for certain use if validated, verified, or reviewed. This may result in updates becoming more frequent, at least concerning the exchange of data where changes are significant.

Source and quality of information It is important to know where information is from and how it is technically validated. Unfortunately, just because information is cited from literature sources or publications does not guarantee a quality seal in terms of its technical content, even if the scientific background and framework is acceptable. A technical proof is assumed when professional review procedures are installed in journal publications - such as here in the International Journal of LCA-and the editors in charge can involve technically knowledgeable reviewers with practical background experience. However if a user aims to produce results and conclusions of practical relevance, taking published information from scientific journals does not release them from their personal responsibility to ensure that the published data sits on adequate primary sources that is relevant to their specific goal. ${ }^{9}$

Transparency of the information source is critical. The data sources must be transparently outlined, e.g., from which companies, associations /member companies, or engineering consultants or engineering institutes it comes from. This is important to facilitate contact to the organizations in case further supportive information is required and to judge the data adequately. Further, it should be clear whether primary data is acquired from running operations with a significant production volume or if it is based on technical reference, design, or planning data.

Another important aspect is the transparency of data representativeness. Is the data covering a representative technology or technology (market or production) mix, a representative production volume, time frame, and region? Supply chain and technology domain expertise is needed to

\footnotetext{
${ }^{9}$ Remark: To support the validation and justification in academia, it is from the authors' perspective - not mandatory to always use "best practice" data to validate new scientific methods. In contrast, it is of utmost importance to use "best practice" data if the aim is to produce results and conclusions towards products, processes or services, and related decisions or measures.
}

qualify (unit process) data and supply chains to be adequate, realistic, and correct.

Not any upstream or precursor unit process step fits to each downstream production step. Country, technology, process improvement, product quality, market, or use situation often define distinct value chains or production mixes of commodities. Where appropriate validation of the supply chain is lacking, unintentional misuse and/or misinterpretation of results is possible, even likely. Verification by industry or certification-organizations with technology domain expertise and respective documentation reduces this danger.

The responsibility for data as well as the ability to reproduce, disclose, and explain data from the data provider (or data compiler) is crucial in professional data supply. The consistent communication of data to an internal or external audience is equally important. If key decisions are made on the basis of applied LCA, e.g., process or product design decisions, it is important for the user to know who is responsible for the data and whom to contact in order to provide additional back-up when important decisions are to be made on the basis of the LCA results.

As for dataset validation, a lot will depend on the effective collaboration between qualified dataset developers (conducting and documenting software-supported plausibility checks during dataset generation) and qualified reviewers validating such development notes and documentation to ascertain whether data quality requirements (temporal, geographical, technological representativeness, completeness, precision, and methodological consistency) have been fulfilled.

Goal-oriented and case-specific approach Probably the first three questions to be answered before planning and applying LCA in industry are:

1. When are the results needed? Tomorrow afternoon or in 18 months?

2. To whom will the results be communicated? To internal experts, management, customers, governmental organizations, NGOs, or the general public?

3. Are all the information needed available internally? Or is external support needed?

These questions are important additions to the structured "goal and scope"-thinking in the ISO standards. In practice, you often need an approximate answer very soon, and a detailed fully validated one within a certain period of time, i.e., a funnel-like escalation of detail. This requires flexible not rigid procedures - also in quality assurance. The answers to the questions above determine what approach, information, data, and reporting is needed to provide timely information, with the appropriate quality and within a defined budget. In summary, the intended application, which is 
per definition beyond the ISO standard, is an important key factor when LCA is to be brought into practice. An LCA practitioner strives to provide answers that are not too complicated by nature, acknowledging relevancy (or nonrelevancy) of specific aspects, focusing on the core parameters, systems and issues and have as much precision as needed to get good answers while avoiding irrelevant complexity. In practice, LCA results produced in-time that point $>80 \%$ in the right direction are improving applications whereas a " $100 \%$ " solution, which can't stick to any deadline does not have any impact in the real world.

Any goal and scope in practice needs its case-specific and responsibly chosen approach and framework. Assessments must be done at the right level, knowing the relevance of the whole life cycle. For example, arguing on the basis of material choice and manufacturing phase scenarios or on other single phases like use phase or end-of-life may be appropriate, but only if one has a good understanding of the environmental performance and sensitivity of all life cycle phases.

Equalization in LCA is often discussed as some stakeholders find ISO standards "imprecise" and call for more rules and a "cookery book" approach. Using LCAs automatically implies accountability and a lack of user responsibility cannot be compensated with rigid rules. Restricting the possibilities reduces useful applications, information, and result exchange, learning curves, and communication of LCA and its (positive) impact overall.

LCAs are often as individual as a diagnosis from a physician. Would you like to see a doctor applying a "medical encyclopedia" in your consultation rather than to check your individual body and come to an individual diagnosis and cure for you? Similarly, health professionals exchange individual results and information while keeping confidentiality and patients profit because the health professionals can decide which approach fits best.

Harmonization and standardization are useful to help to identify those in the business whose intent is to produce and communicate misleading results. LCA is not here to nominate winners, but rather to understand relevant differences, to inform possible trade-offs and to ensure unexpected consequences are minimized. LCA does not aim to announce "absolute" best performers, but rather to indicate individual potentials and relevancy of options. LCA is not a "religion" to tell what is good or bad, it is a tool for finding out what makes sense and what does not. Standardization is highly appreciated in practice. The ISO 14040 series is a core principle and important to prevent LCA "abuse". Further validation or review procedures with meaningful background reports are essential to document the systems, data and results and their transferability or non-transferability towards comparable or other situations.

LCA is a very important "tool in a toolbox" next to others tools like risk assessment and management systems, which is used in companies or organizations to examine a broader range of sustainability impacts of products on society and the natural environment.

\section{No attempt to conclude but to invite}

Historically, the methodological development of LCA has been largely driven by academia striving for innovations to describe the system appropriately. Any changing results of assessments were often perceived in an academic environment as a matter of progress, which is true as long as the assessments do not influence real products and processes. Today, changing results must first be justified in practice before influencing costly redesign or process changes. The strength of LCA is to support many different decisions concerning environmental sustainability in practice in a reliable unambiguous way. As such, LCA as a business case has many valuable facets and adds value to many products and processes. In the last two decades, the perception was mainly that practice should learn from theory and apply new aspects as efficiently and as quickly as possible. It is now time to enter a phase where both academia and industry take the time to begin learning from each other for the sake of strong applied science and successful business applications.

This is nothing new, scientists, engineers, and business leaders in other fields have been doing this for centuries. Take the work of Michael Faraday: It was pure theory until it was picked up by James Clerk Maxwell and put into a suitable mathematical model. Then it was carefully applied by Werner von Siemens in practice and incorporated into products. Adapting this basic approach could indeed lead to a perfect harmony of "ebony and ivory" in the theory and practice of LCA.

We invite everybody for feedback. Any constructive input, comment, or opinion is welcome. Further, we encourage discussions on the role of governments and governmental use of LCA.

Open Access This article is distributed under the terms of the Creative Commons Attribution License which permits any use, distribution, and reproduction in any medium, provided the original author(s) and the source are credited. 